\title{
Condições de atmosfera controlada para pêssegos “Maciel” colhidos em dois estádios de maturação
}

\author{
Controlled atmosphere storage for 'Maciel' peaches harvested in two ripening stages
}

\author{
Ivan Sestari ${ }^{\text {I }}$ Ricardo Fabiano Hettwer Giehl ${ }^{\text {II }}$ Josuel Alfredo Vilela Pinto ${ }^{\text {II }}$ \\ Auri Brackmann"
}

\section{RESUMO}

$O$ objetivo deste trabalho foi avaliar o efeito de duas temperaturas e condições de atmosfera controlada (AC) sobre a conservação de pêssegos da cultivar "Maciel", colhidos em dois estádios de maturação. Os tratamentos avaliados foram: armazenamento refrigerado (AR) na temperatura de $+0,5^{\circ} \mathrm{C} ; \mathrm{AR}$ na temperatura de $-0,5^{\circ} \mathrm{C} ; 2,0 \mathrm{kPa} \mathrm{O}_{2}+4,0 \mathrm{kPa} \mathrm{CO}$ em $-0,5^{\circ} \mathrm{C}$; $1,0 \mathrm{kPaO} \mathrm{O}_{2}+3,0 \mathrm{kPaCO}$ em $-0,5^{\circ} \mathrm{C} ; 2,0 \mathrm{kPaO} \mathrm{O}_{2}+6,0 \mathrm{kPa} \mathrm{CO}_{2}$ em $-0,5^{\circ} \mathrm{C}$. As avaliações foram realizadas após 60 dias de armazenamento e mais dois e quatro dias de exposição dos frutos à temperatura de $20^{\circ} \mathrm{C}$. Na análise realizada após dois meses de armazenamento, mais dois dias a $20^{\circ} \mathrm{C}$, verificou-se que os frutos submetidos a 2,0kPa de $\mathrm{O}_{2}+4,0 \mathrm{kPa}$ de $\mathrm{CO}_{2}$ apresentaram maior firmeza de polpa em relação aos demais tratamentos, sendo que a mesma não foi influenciada pelo estádio de maturação. Os sólidos solúveis totais foram maiores em frutos com estádio de maturação maduro independente da condição de armazenamento. A ocorrência de podridões e escurecimento interno da polpa não foi influenciada pelo estádio de maturação. No entanto, a condição de AC de 1,0 kPa de $\mathrm{O}_{2}+3,0 \mathrm{kPa}$ de $\mathrm{CO}_{2}$ proporcionou o menor percentual de podridões e escurecimento interno da polpa em relação aos demais tratamentos. $\mathrm{Na}$ avaliação realizada aos quatro dias de exposição a $20^{\circ} \mathrm{C}$, os frutos colhidos no estádio maduro estavam completamente podres, independente da condição de armazenamento praticada.

Palavras-chave: Prunus persica, polpa amarela, armazenamento, temperatura, escurecimento interno da polpa.

\section{ABSTRACT}

The objective of this research was to evaluate the effect of temperature and controlled atmosphere (CA) storage conditions on the quality of 'Maciel' peaches harvested in two ripening stages. The treatments were: refrigerated air storage (RA) at $+0.5^{\circ} \mathrm{C} ; \mathrm{RA}$ at $-0.5^{\circ} \mathrm{C} ; 2.0 \mathrm{kPaO} \mathrm{O}_{2}+4.0 \mathrm{kPa} \mathrm{CO}$ plus $-0.5^{\circ} \mathrm{C}$;

\begin{abstract}
$1.0 \mathrm{kPaO} \mathrm{O}_{2}+3.0 \mathrm{kPaCO}$ plus $-0.5^{\circ} \mathrm{C} ; 2.0 \mathrm{kPaO} \mathrm{O}_{2}+6.0 \mathrm{kPa} \mathrm{CO}_{2}$ plus $-0.5^{\circ} \mathrm{C}$. Evaluations of fruit quality were carried out after two months of storage plus two and four days at $20^{\circ} \mathrm{C}$. Fruits stored in $2.0 \mathrm{kPaO} \mathrm{O}_{2}+4.0 \mathrm{kPa} \mathrm{CO}_{2}$ at $-0.5^{\circ} \mathrm{C}$ showed the highest firmness independent of ripening stage. The solid soluble content was higher at mature stage independent of storage conditions. Occurrence of decay and internal browning were not influenced by ripening stage. However, the CA storage at $1.0 \mathrm{kPaO} \mathrm{O}_{2}+3.0 \mathrm{kPaCO}$ plus $-0.5^{\circ} \mathrm{C}$ provided the small occurrence of decay and internal browning of flesh pulp. After four days at $20^{\circ} \mathrm{C}$ fruits at mature stage were completely rotten, independently of storage condition.
\end{abstract}

Key words: Prunus persica, yellow flesh pulp, storage, temperature, internal browning.

\section{INTRODUÇÃO}

Pêssegos da cultivar "Maciel” apresentam dupla finalidade, destinando-se tanto ao consumo in natura quanto ao processamento industrial (CERETTA, 1999). A cultivar apresenta frutos de formato redondocônico e de tamanho grande, com peso médio de $120 \mathrm{~g}$ com película de coloração amarelo-ouro e polpa amarela, não-fundente, firme e aderente ao caroço. Ela destacase pela produtividade, pelo tamanho, pela aparência e pela resistência ao transporte. Além disso, a colheita dos frutos é tardia, sendo realizada no fim de dezembro e início de janeiro, o que abre uma grande possibilidade de exportá-los para os países da Europa, justamente quando estes se encontram em período de entressafra.

O pêssego, devido ao seu acelerado metabolismo no período pós-colheita (COELHO, 1994),

'Departamento de Ciências Biológicas, Escola Superior de Agricultura ‘Luiz de Queiroz’ (ESALQ), Universidade de São Paulo (USP), 13419-140, Piracicaba, SP, Brasil. E-mail: isestari@esalq.usp.br. Autor para correspondência.

IIDepartamento de Fitotecnia, Universidade Federal de Santa Maria (UFSM), Santa Maria, RS, Brasil. 
é altamente perecível, necessitando, dessa forma, de uma rápida comercialização ou de um sistema de armazenamento adequado para chegar em boas condições ao mercado consumidor. O rápido amadurecimento, responsável pelo curto período de prateleira dos frutos, representa sérias restrições ao seu manuseio e transporte (BONGHI et al., 1999).

Na maioria dos casos, a colheita dos frutos é realizada em estádios iniciais de maturação com objetivo de prolongar o período de armazenamento. Em conseqüência, a qualidade sensorial dos frutos é baixa quando estas amadurecem e a susceptibilidade a distúrbios fisiológicos e danos mecânicos aumenta (ROMBALDI et al., 2002; FERRER et al., 2005). Por outro lado, a colheita tardia dos frutos resulta em pêssegos com elevada qualidade, porém eles apresentam baixo potencial de armazenamento, sendo mais apropriados ao consumo imediato (ROMBALDI et al., 2002).

$\mathrm{O}$ armazenamento refrigerado é o principal método utilizado para diminuir o metabolismo e evitar a rápida deterioração de pêssegos. Porém, em armazenamentos mais prolongados, a acentuada perda de firmeza de polpa (SONEGO et al., 1995), a ocorrência de distúrbios fisiológicos (LURIE, 1993; LUCHSINGER et al., 1996) e a incidência de podridões (CERETTA, 1999) são as principais causas de perdas. Além da refrigeração, outras técnicas, como a absorção de etileno e o armazenamento em atmosfera controlada, podem prolongar a conservação e reduzir perdas.

O armazenamento em atmosfera controlada é muito utilizado comercialmente no Brasil para o armazenamento de maçã e kiwi. Para frutas de caroço, a atmosfera controlada é utilizada principalmente para o transporte de frutas do Chile e da África do Sul para os EUA e Europa (BRACKMANN et al., 2003). A redução dos níveis de $\mathrm{O}_{2}$ e o incremento dos níveis de $\mathrm{CO}_{2}$ retardam o amadurecimento dos frutos, reduzem a síntese e a ação do etileno sobre o metabolismo e a ocorrência de podridões (KE et al., 1991; BRACKMANN \& CHITARRA, 1998).

As pressões parciais mínimas de $\mathrm{O}_{2}$ e máximas de $\mathrm{CO}$ variam de acordo com a sensibilidade dos tecidos à indução da respiração anaeróbia e injúria causada pelo $\mathrm{CO}_{2}$, respectivamente, sendo estas características variáveis entre as diferentes cultivares (SALVADOR et al., 1998; JIANG et al., 2002). Segundo KADER (1989), pêssegos toleram pressão parcial mínima de $\mathrm{O}_{2}$ e máxima de $\mathrm{CO}_{2}$ de $2 \mathrm{kPa} \mathrm{O}_{2}$ e $5 \mathrm{kPa} \mathrm{CO}_{2}$, respectivamente. ERIS et al. (1994) obtiveram melhor conservação de pêssegos com $2 \mathrm{kPa} \mathrm{O}_{2}$ e com $5 \mathrm{kPa}$ ou $10 \mathrm{kPa} \mathrm{CO}_{2}$. Por outro lado, CERETTTA et al. (2000) concluíram que a melhor condição de AC para o pêssego “Eldorado” é $1 \mathrm{kPa} \mathrm{O}_{2}$ e $3 \mathrm{kPa} \mathrm{CO}_{2}$.
Tendo em vista a possibilidade de exportação de pêssegos de polpa amarela para a Europa por meio do uso do transporte marítimo, há necessidade de gerar tecnologia de armazenamento que permita a conservação da qualidade dos frutos. Portanto, este trabalho tem o objetivo de avaliar o efeito de duas temperaturas e condições de atmosfera controlada (AC) sobre a conservação de pêssegos da cultivar Maciel, colhidos em dois estádios de maturação, visando ao transporte de longa distância.

\section{MATERIAL E MÉTODOS}

O experimento foi conduzido no Núcleo de Pesquisa em Pós-Colheita (NPP) do Departamento de Fitotecnia da Universidade Federal de Santa Maria, RS. Foram utilizados frutos da cultivar Maciel provenientes de um pomar comercial localizado no município de Canguçú, RS. O delineamento experimental utilizado foi o inteiramente casualizado com cinco tratamentos e quatro repetições de 33 frutos cada, sendo estas compostas por frutos em dois estádios de maturação na colheita (maduro e verdoengo). As condições de armazenamento avaliadas foram: armazenamento refrigerado (AR) na temperatura de $+0,5^{\circ} \mathrm{C}$; $\mathrm{AR}$ na temperatura de $-0,5^{\circ} \mathrm{C} ; 2,0 \mathrm{kPa} \mathrm{O}+4,0 \mathrm{kPa} \mathrm{CO}_{2}$ em $-0,5^{\circ} \mathrm{C} ; 1,0 \mathrm{kPa} \mathrm{O}_{2}+3,0 \mathrm{kPa} \mathrm{CO}_{2} \mathrm{em}^{-0}, 5^{\circ} \mathrm{C} ; 2,0 \mathrm{kPa} \mathrm{O}_{2}+$ $6,0 \mathrm{kPaCO} \mathrm{em}^{2} 0,5^{\circ} \mathrm{C}$.

$\mathrm{O}$ armazenamento foi realizado em minicâmaras experimentais de atmosfera controlada, com volume de 232L. O monitoramento da temperatura foi realizado diariamente utilizando termômetros de mercúrio introduzidos na polpa de frutos. A umidade relativa (UR) no interior das minicâmaras de atmosfera controlada foi mantida em torno de 95\%, sendo aferida semanalmente com auxílio de um psicrômetro.

A manutenção das pressões parciais desejadas dos gases nas câmaras, que variavam em função da respiração dos frutos, foi feita por meio da análise diária com uso de analisadores eletrônicos de $\mathrm{CO}_{2}$ e $\mathrm{O}_{2}$, marca Agri-datalog, e posterior correção das pressões parciais até os níveis pré-estabelecidos nos tratamentos. $\mathrm{O} \mathrm{O}_{2}$ consumido pela respiração foi reposto por meio da injeção de ar atmosférico nas minicâmaras. $\mathrm{O} \mathrm{CO}_{2}$ em excesso foi absorvido por uma solução de hidróxido de potássio (40\%), por meio da qual foram circulados os gases das minicâmaras.

A avaliação do experimento foi realizada após 60 dias de armazenamento mais dois e quatro dias de exposição dos frutos à temperatura de $20^{\circ} \mathrm{C}$, simulando o período de comercialização. Foram avaliadas as seguintes variáveis: firmeza de polpa, determinada com o uso de penetrômetro manual com 
ponteira de 7,8mm de diâmetro; acidez titulável (AT), avaliada por meio de titulação de $10 \mathrm{~mL}$ de suco em $100 \mathrm{~mL}$ de água destilada com solução de $\mathrm{NaOH}$ a $0,1 \mathrm{~N}$ até $\mathrm{pH}$ 8,1; sólidos solúveis totais (SST), determinados com o uso de refratômetro manual com correção da temperatura; cor de fundo da epiderme, medida por meio de um colorímetro, marca Minolta, modelo CR 310, com os resultados expressos em ângulo de cor $\left(\mathrm{h}^{\circ}\right)$. O $\mathrm{h}^{\circ}$ define a coloração básica, sendo que $0^{\circ}=$ vermelho; $90^{\circ}=$ amarelo, $180^{\circ}=$ verde (McGUIRE, 1992); ocorrência de podridões, obtida pelo percentual de frutos com lesões de diâmetro igual ou superior a $0,5 \mathrm{~cm}$, com características de ataques de patógenos. O escurecimento interno da polpa foi determinado por meio da contagem de frutos com qualquer tipo de escurecimento interno na polpa. Os dados obtidos foram submetidos à análise de variância e as médias comparadas pelo teste de Duncan a 5\% de probabilidade de erro, sendo que os dados em porcentagem foram transformados por meio da fórmula arco-seno $\sqrt{\mathrm{x} / 100}$.

\section{RESULTADOS E DISCUSSÃO}

Após dois meses de armazenamento em atmosfera controlada, mais dois dias a $20^{\circ} \mathrm{C}$, os frutos submetidos a $2,0 \mathrm{kPa} \mathrm{O}_{2}+4,0 \mathrm{kPa} \mathrm{CO}_{2}$ na temperatura de $-0,5^{\circ} \mathrm{C}$ apresentaram maior firmeza de polpa em relação aos demais tratamentos, sendo que a mesma não foi influenciada pelo estádio de maturação (Tabela 1). Resultados similares foram obtidos por NAVA \& BRACKMANN (2002). A maior firmeza de polpa verificada no tratamento com $2,0 \mathrm{kPa} \mathrm{O}_{2}+4,0 \mathrm{kPa} \mathrm{CO}_{2}$ na temperatura de $-0,5^{\circ} \mathrm{C}$ pode estar relacionada a um maior decréscimo na atividade das enzimas endoglucanase, poligalacturonase e pectinametilesterase que atuam na degradação da parede celular (BONGHI et al., 1999; ORR \& BRADY, 1993; FERNÁNDEZTRUJILLO et al., 1998).

Os sólidos solúveis totais (SST) foram maiores nos frutos colhidos no estádio de maturação maduro independente da condição de armazenamento (Tabela 2). De acordo com KE et al. (1991), o conteúdo de SST sofre pequenas variações durante o armazenamento, sendo que este parâmetro não é influenciado pela temperatura e concentração de oxigênio. Nesse sentido, MURRAY \& VALENTINI (1998) e ROMBALDI et al. (2002) sugerem que, para frutas de caroço, o incremento de SST após a colheita está relacionado com o ponto de colheita dos frutos e a qualidade do sistema de armazenamento.

O armazenamento em atmosfera controlada, independente da pressão parcial de gases utilizada, propiciou maior conteúdo de acidez titulável em relação ao armazenamento refrigerado na avaliação realizada após o período de armazenamento mais dois dias a $20^{\circ} \mathrm{C}$ para os frutos colhidos no estádio de maturação verdoengo (Tabela 2). O efeito da atmosfera controlada na redução da degradação dos ácidos possivelmente esteja relacionado ao efeito inibitório do $\mathrm{CO}_{2}$ sobre as enzimas aconitase, succinato desidrogenase e isocitrato desidrogenase do ciclo dos ácidos tricarboxílicos (BRACKMANN et al., 2003).

Em virtude do prolongado período de armazenamento, na avaliação dos frutos após dois dias de exposição a $20^{\circ} \mathrm{C}$, verificou-se elevado percentual de podridão nos frutos submetidos ao armazenamento refrigerado e nas condições de $\mathrm{AC}$ com $2 \mathrm{kPa}$ de $\mathrm{O}_{2}$ (Tabela 3). Por outro lado, o tratamento com 1,0 kPa de

Tabela 1 - Firmeza de polpa de pêssegos cv. "Maciel” após dois meses de armazenamento refrigerado ou em atmosfera controlada mais dois dias a $20^{\circ}$ C. Santa Maria, RS. 2004.

\begin{tabular}{|c|c|c|c|c|}
\hline \multirow{2}{*}{ Temperatura ${ }^{\circ} \mathrm{C}$} & \multirow{2}{*}{ Condições de armazenamento $\mathrm{O}_{2}+\mathrm{CO}_{2}(\mathrm{kPa})$} & \multicolumn{2}{|c|}{----------Estádio de maturação---------- } & \multirow{2}{*}{ Média } \\
\hline & & Maduros & Verdoengo & \\
\hline $0,5^{\circ} \mathrm{C}$ & $\mathrm{AR}^{* *}$ & $41,2 *$ & 36,9 & $39,1 \mathrm{c}$ \\
\hline$-0,5^{\circ} \mathrm{C}$ & AR & 38,4 & 35,8 & $37,1 \mathrm{c}$ \\
\hline$-0,5^{\circ} \mathrm{C}$ & $2,0+4,0$ & 43,0 & 46,4 & $44,7 a$ \\
\hline$-0,5^{\circ} \mathrm{C}$ & $1,0+3,0$ & 40,3 & 42,5 & $41,4 b$ \\
\hline$-0,5^{\circ} \mathrm{C}$ & $2,0+6,0$ & 35,9 & 38,2 & $37,0 \mathrm{c}$ \\
\hline Média & & $39,7 \mathrm{~A}$ & $39,9 \mathrm{~A}$ & \\
\hline CV (\%) & & & & \\
\hline
\end{tabular}

*Tratamentos com médias seguidas pela mesma letra, maiúscula na horizontal e minúscula na vertical, diferem pelo teste de Duncan a 5 \% de probabilidade de erro.

** AR- armazenamento refrigerado. 
Tabela 2 - Teor de sólidos solúveis totais e acidez titulável em pêssegos cv. "maciel” após dois meses de armazenamento refrigerado ou em atmosfera controlada mais dois dias a $20^{\circ} \mathrm{C}$. Santa Maria, RS. 2004.

\begin{tabular}{|c|c|c|c|c|c|c|c|}
\hline \multirow{3}{*}{ Temperatura ${ }^{\circ} \mathrm{C}$} & \multirow{3}{*}{$\begin{array}{l}\text {-Tratamentos--------------- } \\
\text { Condições de armazenamento } \\
\qquad \mathrm{O}_{2}+\mathrm{CO}_{2}(\mathrm{kPa})\end{array}$} & \multicolumn{3}{|c|}{------Sólidos solúveis totais ( ${ }^{\circ}$ Brix)------ } & \multicolumn{3}{|c|}{------Acidez titulável $\left(\mathrm{cmol} . \mathrm{L}^{-1}\right)-----$} \\
\hline & & \multicolumn{2}{|c|}{ Estádio de maturação } & \multirow{2}{*}{ Média } & \multicolumn{2}{|c|}{ Estádio de maturação } & \multirow{2}{*}{ Média } \\
\hline & & Maduros & Verdoengo & & Maduros & Verdoengo & \\
\hline $0,5^{\circ} \mathrm{C}$ & $\mathrm{AR}^{* *}$ & 11,3 & 11,0 & $11,1 \mathrm{a}$ & 5,3 & 5,9 & $5,6 c$ \\
\hline$-0,5^{\circ} \mathrm{C}$ & AR & 11,8 & 10,5 & $11,1 \mathrm{a}$ & 5,6 & 7,1 & $6,4 \mathrm{~b}$ \\
\hline$-0,5^{\circ} \mathrm{C}$ & $2,0+4,0$ & 11,8 & 11,2 & $11,5 a$ & 7,0 & 8,9 & $8,0 a$ \\
\hline$-0,5^{\circ} \mathrm{C}$ & $1,0+3,0$ & 11,4 & 10,7 & $11,0 \mathrm{a}$ & 7,8 & 9,1 & $8,5 a$ \\
\hline$-0,5^{\circ} \mathrm{C}$ & $2,0+6,0$ & 11,5 & 10,9 & $11,2 \mathrm{a}$ & 7,5 & 8,5 & $8,0 \mathrm{a}$ \\
\hline Média & & $11,5 \mathrm{~A}$ & $10,8 \mathrm{~B}$ & & $6,6 \mathrm{~B}$ & 7,9A & \\
\hline CV (\%) & & \multicolumn{2}{|c|}{2,37} & & \multicolumn{2}{|c|}{6,02} & \\
\hline
\end{tabular}

*Tratamentos com médias seguidas pela mesma letra, maiúscula na horizontal e minúscula na vertical, diferem pelo teste de Duncan a 5 \% de probabilidade de erro.

** AR- armazenamento refrigerado.

$\mathrm{O}_{2}$ demonstrou ser efetivo no controle de podridões, alcançando percentual de incidência inferior a 10\%. De acordo com BRACKMANN \& CHITARRA (1998), a atmosfera controlada exerce grande efeito na redução da incidência de podridões, que se dá por meio do retardamento da degradação de pectinas da parede celular, tornando o fruto mais resistente ao ataque de fungos. Já após quatro dias de exposição dos frutos a $20^{\circ} \mathrm{C}$, os frutos colhidos no estádio de maturação maduros apresentaram podridão na totalidade dos frutos, independente da condição de armazenamento, evidenciando a alta sensibilidade da cultivar à podridão parda (Tabela 3). Mesmo com o uso de AC, a incidência de podridões foi superior a 90\%, em decorrência do prolongado período de armazenamento e da alta perecibilidade dos frutos durante o período de prateleira.

Semelhante ao verificado para a ocorrência de podridão, o escurecimento interno da polpa não foi influenciado pelo estádio de maturação dos frutos na colheita (Tabela 4). A menor ocorrência de escurecimento interno ocorreu na condição de AC com $1,0 \mathrm{kPa} \mathrm{O}_{2}+3,0 \mathrm{kPa} \mathrm{CO}_{2}$, porém, não diferindo estatisticamente das demais condições de AC. Contrariamente, na cultivar Chiripá a ocorrência de escurecimento da polpa somente foi observada após

Tabela 3 - Ocorrência de podridões em pêssegos cv. "maciel” após dois meses de armazenamento refrigerado ou atmosfera controlada mais dois e quatro dias a $20^{\circ} \mathrm{C}$. Santa Maria, RS. 2004.

\begin{tabular}{|c|c|c|c|c|c|c|c|}
\hline \multirow{3}{*}{ Temperatura ${ }^{\circ} \mathrm{C}$} & \multirow{3}{*}{$\begin{array}{l}\text { Condições de armazenamento } \\
\qquad \mathrm{O}_{2}+\mathrm{CO}_{2}(\mathrm{kPa})\end{array}$} & \multicolumn{3}{|c|}{-----------------2 dias----------------- } & \multicolumn{3}{|c|}{------------------4 dias----------------- } \\
\hline & & \multicolumn{2}{|c|}{ Estádio de maturação } & \multirow{2}{*}{ Média } & \multicolumn{2}{|c|}{ Estádio de maturação } & \multirow{2}{*}{ Média } \\
\hline & & Maduros & Verdoengo & & Maduros & Verdoengo & \\
\hline $0,5^{\circ} \mathrm{C}$ & $\mathrm{AR}^{* *}$ & $80,0^{*}$ & 97,3 & $88,6 a$ & $100,0 *$ & 100,0 & $100,0 \mathrm{a}$ \\
\hline$-0,5^{\circ} \mathrm{C}$ & $\mathrm{AR}$ & 80,7 & 63,1 & 71,9ab & 100,0 & 96,1 & $98,0 \mathrm{ab}$ \\
\hline$-0,5^{\circ} \mathrm{C}$ & $2,0+4,0$ & 43,9 & 68,4 & $56,1 b$ & 100,0 & 98,4 & $99,2 \mathrm{ab}$ \\
\hline$-0,5^{\circ} \mathrm{C}$ & $1,0+3,0$ & 10,6 & 8,3 & $9,2 \mathrm{c}$ & 100,0 & 83,8 & $91,8 b$ \\
\hline$-0,5^{\circ} \mathrm{C}$ & $2,0+6,0$ & 59,9 & 78,9 & 69,4ab & 100,0 & 93,9 & $96,9 a b$ \\
\hline Média & & $55,0 \mathrm{~A}$ & $63,1 \mathrm{~A}$ & & $100,0 \mathrm{~A}$ & $94,4 \mathrm{~B}$ & \\
\hline CV (\%) & & & & & & & \\
\hline
\end{tabular}

*Tratamentos com médias seguidas pela mesma letra, maiúscula na horizontal e minúscula na vertical, diferem pelo teste de Duncan a 5 \% de probabilidade de erro.

** AR- armazenamento refrigerado.

Ciência Rural, v.38, n.5, ago, 2008. 
Tabela 4 - Percentual de escurecimento interno da polpa e coloração da casca de pêssegos cv. “maciel” após dois meses de armazenamento refrigerado ou em atmosfera controlada mais dois dias a $20^{\circ} \mathrm{C}$. Santa Maria, RS. 2004.

\begin{tabular}{|c|c|c|c|c|c|c|c|}
\hline \multicolumn{2}{|c|}{ 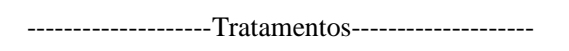 } & \multicolumn{3}{|c|}{-------Escurecimento interno (\%)-------- } & \multicolumn{3}{|c|}{ 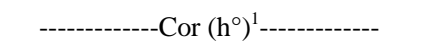 } \\
\hline \multirow{2}{*}{ Temperatura ${ }^{\circ} \mathrm{C}$} & \multirow{2}{*}{$\begin{array}{l}\text { Condições de armazenamento } \\
\qquad \mathrm{O}_{2}+\mathrm{CO}_{2}(\mathrm{kPa})\end{array}$} & \multicolumn{2}{|c|}{ Estádio de maturação } & \multirow{2}{*}{ Média } & \multicolumn{2}{|c|}{ Estádio de maturação } & \multirow{2}{*}{ Média } \\
\hline & & Maduros & Verdoengo & & Maduros & Verdoengo & \\
\hline $0,5^{\circ} \mathrm{C}$ & $\mathrm{AR}^{* *}$ & 100,0 & 87,5 & $93,7 a$ & 82,2 & 83,2 & $82,7 \mathrm{~b}$ \\
\hline$-0,5^{\circ} \mathrm{C}$ & $\mathrm{AR}$ & 63,1 & 81,2 & $72,2 \mathrm{ab}$ & 85,6 & 89,3 & $87,4 a$ \\
\hline$-0,5^{\circ} \mathrm{C}$ & $2,0+4,0$ & 78,7 & 13,1 & $45,9 \mathrm{bc}$ & 80,4 & 84,1 & $82,2 \mathrm{~b}$ \\
\hline$-0,5^{\circ} \mathrm{C}$ & $1,0+3,0$ & 28,7 & 26,6 & $27,7 \mathrm{c}$ & 80,3 & 84,2 & $82,2 \mathrm{~b}$ \\
\hline$-0,5^{\circ} \mathrm{C}$ & $2,0+6,0$ & 44,5 & 40,7 & $42,6 \mathrm{bc}$ & 79,4 & 84,1 & $81,7 \mathrm{~b}$ \\
\hline Média & & $63,0 \mathrm{~A}$ & 49,9A & & $81,5 \mathrm{~B}$ & $85,0 \mathrm{~A}$ & \\
\hline CV (\%) & & & & & & & \\
\hline
\end{tabular}

*Tratamentos com médias seguidas pela mesma letra, maiúscula na horizontal e minúscula na vertical, diferem pelo teste de Duncan a 5 \% de probabilidade de erro.

** AR- armazenamento refrigerado.

1. ângulo hue $\mathrm{h}^{\circ}\left(0^{\circ}=\right.$ vermelho; $90^{\circ}=$ amarelo; $180^{\circ}=$ verde; $360^{\circ}=$ azul $)$

dois dias a $20^{\circ} \mathrm{C}$ em frutos armazenados em ar refrigerado (BRACKMANN et al., 2003). Este resultado pode ser explicado pela presença de grande concentração de fenóis totais na polpa da cultivar Maciel, em relação a outras cultivares, os quais estão relacionados intimamente com o escurecimento enzimático, pois são substrato para a polifenoloxidase (TORALLES et al., 2004).

Os frutos colhidos no estádio de maturação verdoengo mantiveram a coloração de fundo da epiderme mais verde em relação aos frutos colhidos maduros. Embora sem diferença estatística, os frutos armazenados em AC permaneceram com coloração da epiderme mais verde que os armazenados sob refrigeração na temperatura de $-0,5^{\circ} \mathrm{C}$ (Tabela 4). Resultado semelhante foi observado por NAVA \& BRACKMANN (2002), que atribuem a manutenção da coloração verde da epiderme devido ao efeito da AC sobre a atividade das clorofilases.

Assim, sugerem-se investigações adicionais no sentido de melhorar o controle fitossanitário em pré-colheita para que se consiga transportar pêssegos "Maciel" via marítima num período de três semanas sem grandes perdas decorrentes de podridões.

\section{CONCLUSÕES}

A melhor condição para o armazenamento de pêssegos "Maciel" é na temperatura de $-0,5^{\circ} \mathrm{C}$, associada com o uso de atmosfera controlada com $1,0 \mathrm{kPa} \mathrm{O}_{2}+3,0 \mathrm{kPa} \mathrm{CO}$. Frutos colhidos com estádio de maturação verdoengo apresentam teor de sólidos solúveis totais suficientes para uma boa qualidade e conserva-se melhor que frutos maduros.
Deve-se evitar armazenar pêssegos 'Maciel' durante dois meses na temperatura de $0,5^{\circ} \mathrm{C}$, pois os frutos apresentaram alta percentagem de podridão e escurecimento interno, quando expostos por mais de dois dias a $20^{\circ} \mathrm{C}$.

\section{REFERÊNCIAS}

BONGHI, C. et al. Peach fruit ripening and quality in relation to picking time, and hypoxic and high $\mathrm{CO}_{2}$ short-term postharvest treatments. Postharvest Biology and Technology, v.16, n.3, p.213-222, 1999.

BRACKMANN, A.; CHITARRA, A.B. Atmosfera controlada e atmosfera modificada. In:______. Armazenamento e processamento de produtos agrícolas. Lavras: UFLA/ SBEA, 1998. p.133-170.

BRACKMANN, A. et al. Armazenamento de pêssego Chimarrita em atmosfera controlada e sob absorção de etileno. Ciência Rural, v.33, n.3, p.431-435, 2003.

CERETA, M. Qualidade do pêssego, cv. Eldorado, armazenado em atmosfera controlada. Pelotas - RS. 1999. 46f. Dissertação (Mestrado em Agronomia) - Programa de Pós-graduação em Agronomia, Universidade Federal de Pelotas.

CERETTA, M. et al. Conservação em atmosfera controlada de pêssego Eldorado. Ciência Rural, v.30, n.1, p.73-79, 2000.

COELHO, A.H.R. Qualidade pós-colheita de pêssegos. Informe Agropecuário, v.17, n.180, p.5-9, 1994.

ERIS, A. et al. A research on controlled atmosphere (CA) storage of peach cv. Hale Haven. Acta Horticulturae, v.1 n.368, p.767-776, 1994.

FERNÁNDEZ-TRUJILLO, J.P. et al. Physiological changes in peaches related to chilling injury and ripening. Postharvest Biology and Technology, v.13, n.2, p.109-119, 1998. 
FERRER, A. et al. Changes during the ripening of the very late season Spanish peach cultivar 'Calanda' Feasibility of using CIELAB coordinates as maturity indices. Scientia Horticulturae, v.105, n.4, p.435-446, 2005.

JIANG, A.L. et al. Effects of controlled atmospheres with high- $\mathrm{O}_{2}$ or high $\mathrm{CO}_{2}$ concentrations on postharvest physiology and storability of "Napoleon" sweet cherry. Acta Botanica Sinica, v.44, p.925-930, 2002.

KADER, A.A.; MITCHELL, F.G. Maturity and quality. In Peaches, plums and nectarines - growing and handling for fresh market. Oakland: University of California Division of Agricultural and Natural Resources, 1989. p.191-196.

KE, D.Y. et al. Physiological-responses and quality attributes of peaches kept in low oxygen atmospheres. Scientia Horticulturae, v.47, n.3-4, p.295-303, 1991.

LUCHSINGER, L.E. et al. Chilling injury of peach fruits during storage. Horticulturae Science, v.25, n.5, p.31-36, 1996.

LURIE, S. Modified atmosphere storage of peaches and nectarines to reduce storage disorders. Journal Food Quality v.16, n.1, p.56-65, 1993

McGUIRE, R.G. Reporting of objective color measurements. HortScience, v.27, n.12, p.1254-1255, 1992.
MURRAY, R.; VALENTINI, G. Storage and quality of peach fruit harvest at different stages of maturity. Acta Horticulturae, v.2, n.465, p.455-463, 1998.

NAVA, A.G.; BRACKMANN, A. Armazenamento de pêssegos (Prunus persica (L.) Batsch), cv. Chiripá, em atmosfera controlada. Revista Brasileira de Fruticultura, v.24, n.2, p.328-332, 2002.

ORR, G.; BRADY, C.J. Relationship of endopolygalacturonase activity to fruit softening in a freestone peach. Postharvest Biology and Technology, v.3, n.2, p.121-130, 1993.

ROMBALDI, C.V. et al. Armazenamento de pêssegos (Prunus persica L.), cultivar Chiripá, em atmosfera controlada. Ciência Rural, v.32, n.1, p.43-47, 2002.

SALVADOR, M.E. et al. Locality effect on some fruit quality parameters in peaches and nectarines. Acta Horticulturae, v.2, n.465, p.447-451, 1998.

SONEGO, L. et al. Biochemical and physical evaluation of textural characteristics of nectarines exhibiting woolly breakdown. NMR imaging, X-ray computed tomography and pectin composition. Postharvest Biology and Technology, v.5, n.3, p.187-198, 1995.

TORALLES, R.P. et al. Caracterização parcial do escurecimento enzimático pela polifenoloxidase em pêssegos das cvs. Granada, Jade, Esmeralda e Maciel. Revista Brasileira de Agrociência. v.10, n.1, p.241-244, 2004 